\title{
Does Advice Based on Less Restrictive Sucrose Intake Affect Glycaemic Control in Individuals with Established Type 2 Diabetes?
}

\author{
Jacqueline Cleator ${ }^{1,3}$, Karen Allan ${ }^{2}$, Elizabeth Smith $^{1}$, Gemma Jaggard $^{1}$ and \\ John Wilding ${ }^{1}$ \\ ${ }^{1}$ Department of Obesity \& Endocrinology, University of Liverpool, Liverpool, England, UK \\ ${ }^{2}$ Professional and Practice Development Unit NHS Grampian, Foresterhill House, Aberdeen, UK \\ ${ }^{3}$ School of Nursing, Midwifery \& Social Work, University of Manchester, Jean McFarlane Building, \\ Oxford Road, Manchester, England, UK
}

Correspondence should be addressed to: Jacqueline Cleator; jacqueline.cleator@manchester.ac.uk Received Date: 24 November 2013; Accepted Date: 21 January 2014; Published Date: 29 April 2014

Academic Editor: Kantartzis, Konstantinos

Copyright (c) 2014 Jacqueline Cleator, Karen Allan, Elizabeth Smith, Gemma Jaggard and John Wilding. Distributed under Creative Commons CC-BY 3.0

\begin{abstract}
This 12-month, randomised study of individuals with established type 2 diabetes investigated whether dietary advice emphasising a single message to reduce fat intake, would improve weight loss, glycaemic and lipid control, compared to advice based on the once traditional lowfat, low-sucrose message. Participants $(\mathrm{n}=37$, mean BMI $33.25(+1.0) \mathrm{kg} / \mathrm{m} 2$, age $58.0(+3.3)$ years, diabetes duration $6.8( \pm 0.9)$ yrs, $51 \%$ male), were randomised into 2 dietary groups. The 'moderate-sucrose' group $(\mathrm{n}=19)$ were advised to reduce total fat intake only; the 'low-sucrose' group $(n=18)$, to reduce both total fat and total sugar intake. Repeated measures ANOVA determined group effects on anthropometric/biochemical variables and Spearman's rank correlations determined relationships between changes in diet and study variables. Reported energy and fat intake significantly reduced in both groups, with no difference in reported sucrose intake. HbA1c fell in both groups (7.7\% - 7.1\% (low-sucrose) vs 7.6\% - 7.4\% (moderate-sucrose), both $\mathrm{p}=0.003$ ), with no additional effect of the single dietary message. HOMA-IR fell by $33.4 \%$ in the 'low-sucrose' group ( $\mathrm{p} \leq 0.005)$, but not in the moderate sucrose group. Advice to relax sucrose intake restrictions in favour of a single low fat message had no additional effect on reported behaviour or glycaemic control.
\end{abstract}

Keywords: Type 2 diabetes, sucrose, dietary advice, low fat.

Cite this Article as: Jacqueline Cleator, Karen Allan, Elizabeth Smith, Gemma Jaggard and John Wilding (2014), "Does Advice Based on Less Restrictive Sucrose Intake Affect Glycaemic Control in Individuals with Established Type 2 Diabetes?," Journal of Research in Diabetes, Vol. 2014 (2014), Article ID 224230, DOI: $10.5171 / 2014.224230$ 


\section{Introduction}

Type 2 diabetes is a health problem of epidemic proportions, driven in part by increasing obesity and an ageing population (Mensah et al. 2004). Compared to 2010 levels, it is anticipated that by 2030, numbers of adults with type 2 diabetes will have increased by $69 \%$ in developing countries and by $20 \%$ in developed countries (Shaw, Sicree, \& Zimmet 2010). The link between obesity and type 2 diabetes is well established, as adiposity has been clearly shown to aggravate insulin resistance and its associated cardiovascular risk factors (Mensah et al. 2004). Eighty percent of people are overweight or obese when they are diagnosed with type 2 diabetes (Smyth \& Heron 2006). It is understandable, therefore, given the inextricable link with obesity, that dietary energy restriction and exercise to promote weight loss remain the cornerstones of the management of type 2 diabetes (Dyson et al. 2011).

Diabetes UK (2011) recommend a flexible dietary approach, with a focus on total energy intake rather than macronutrient composition. Foods with a low glycaemic index are actively promoted and saturated fats should be limited and replaced by unsaturated fats (predominantly monounsaturated fats) (Riserus, Willett, \& $\mathrm{Hu} 2009$ ). For those who are overweight or obese, weight loss should be the primary nutritional strategy in managing glucose control (Dyson et al 2011).

For people with longstanding type 2 diabetes, recommendations regarding sucrose have changed over time, moving from a $25 \mathrm{~g}$ daily sucrose restriction proposed by the British Dietetic Association in 1992 (Department of Health 1991) to that of the general UK population guidelines, that $10 \%$ of total dietary intake can be derived from non-milk extrinsic sugars. Yet the imperative for those with type 2 diabetes to avoid sucrose at all costs still persists as an enduring message, familiar to most people, regardless of their disease status. The previous long-standing antipathy to sucrose was reinforced during the 1980 s by a series of studies suggesting that sucrose aggravated hyperglycaemia and dyslipidaemia. However, later reports cast doubt on these findings, with studies including more subjects and of longer duration; sucrose intakes of up to 200 g/day did not demonstrate such deleterious effects and influenced the decision to relax the recommendations (Abraira \& Derler 1988; Bantle, Laine, \& Thomas 1986; Black et al. 2006; Jellish, Emanuele, \& Abraira 1984).

In theory, one would expect that allowing individuals with type 2 diabetes to use sucrose more freely would simplify dietary advice, by removing the need to conform to two 'negative' dietary messages and placing emphasis on one dietary message only, namely a reduction in fat intake. Providing subjects had a clearer understanding of what $10 \%$ of energy as sugar looks like, this would enable them to focus on reducing dietary fat and overall energy intake and moreover, dietary compliance might be enhanced due to improved palatability of the diet.

\section{Study Aims}

The purpose of this study was to test the hypothesis that dietary advice emphasising a single dietary restriction, i.e the reduction of fat, will improve compliance, enhance weight and fat loss, and improve blood glucose, lipid levels and insulin sensitivity, as compared with advice based on the two restrictions contained in the once traditional low-fat, low-sucrose message. The primary endpoint was the change in $\mathrm{HbA1c}$ from baseline to end of study. Secondary endpoints were the change in weight from baseline to end of study and postprandial triglyceride levels.

\section{Research Design and Methodology}

\section{Subjects}

This was a 12-month, randomised study of individuals with type 2 diabetes attending a typical diabetes out-patient facility in the UK. Participants were recruited from the Diabetes Centre at Walton Hospital, Aintree, Liverpool. Only those with a body mass index $(\mathrm{BMI}) \geq 27 \mathrm{~kg} / \mathrm{m}^{2}$ were 
included, to ensure that all received the same hypocaloric advice. In addition, participants were required to be stable on metformin monotherapy only for a minimum of 6 months prior to entry, as clinic data available for subjects on metformin monotherapy showed that weight tended to fall after 3 months of treatment, returning to baseline at 6 months. Similarly, those taking any medication known to affect weight, such as $\beta$-blockers, diuretics or thyroxine had to be stable on therapy for a minimum of 6 months. In order to dilute the impact of previous dietary messages, participants who had had an appointment with a dietitian in the previous 18 months were excluded. Individuals taking lipid-lowering medication were excluded from the test meal arm of the study. South Sefton Research Ethics Committee approved the study (ref no EC.115.99) and written informed consent was obtained from all participants.

\section{Anthropometric and Biochemical Assessments.}

Individuals attended in the morning after an overnight fast and were randomised by the study nurse using sealed envelopes to determine treatment group, to a stringently restricted group (low-sucrose) with strong emphasis on the two messages to reduce both total dietary fat and total sugar intake, or to a moderately restricted advice group (moderate-sucrose), with emphasis on the single message to reduce total fat intake. Body height and weight to calculate BMI and waist circumference were measured at three monthly intervals by the study nurse and lean body fat mass was estimated noninvasively by bioelectrical impedance, a measure of body composition which has been validated favourably against a fourcompartment model (Jebb et al. 2000). Participants' estimated total energy requirements were calculated using the Schofield predictive equations (Schofield 1985). These use age, gender and weight to predict basal metabolic rate, combined with an assessment of an individual's physical activity level (PAL), as set out in the UK daily reference values (Department of Health 1991).Participants were encouraged to monitor their blood glucose levels on one day per week, by measuring fasting and mid-afternoon post-prandial blood glucose concentrations, accepted indices of overall glycaemic control (Avignon, Radauceanu, \& Monnier 1997). Te st-strips and an electrochemical meter (Medisense-Precision) were supplied and monitoring technique was taught and reinforced by the study nurse. Fasting glucose laboratory samples and HbA1c levels were monitored 3-monthly, along with fasting lipid profiles, including triglycerides (TAG), total, LDL- and HDLcholesterol and the total cholesterol:HDL ratio.

As raised postprandial triglyceride levels are known to aggravate cardiovascular risk (Mora et al. 2008), those not taking lipidmodifying medication were given a test breakfast at baseline and 12 months to determine whether dietary advice based on less restrictive sucrose had an adverse effect on lipid profiles. The test meal followed standard methods for determining post prandial lipaemia (van Oostrom et al. 2009) and comprised 729 calories (49.4g CHO, $50 \mathrm{~g}$ fat) made from croissants $(100 \mathrm{~g})$, butter $(10 \mathrm{~g})$, cheese slices $(40 \mathrm{~g})$, whole milk $(100 \mathrm{ml})$ and cocoa powder $(10 \mathrm{~g})$. Venous blood was sampled at baseline and every hour for 5 hours; area under the curve Whilst it is acknowledged that post-prandial hypertriglyceridaemia may take $>9$ hours to clear completely in individuals with type 2 diabetes, 5 hours was felt to be the maximum time tolerable for our participants. Insulin sensitivity was assessed 3-monthly by calculating the HOMA-IR index from fasting insulin and glucose concentrations (Matthews et al. 1985). This generally accepted and validated index is particularly useful for following longitudinal changes in individual insulin sensitivity. The DPC IMMULITE insulin assay (a two-site chemiluminescent enzyme immunometric assay), performed routinely by the hospital biochemistry laboratory was used to measure insulin levels. 


\section{Materials}

After all baseline fasting assessments had been completed and participants had eaten breakfast, dietary advice was delivered by the study dietitian according to randomised group. All participants received advice based on a $500 \mathrm{kcal}$ daily deficit according to their total energy requirements. Written dietary advice was provided in the form of a different booklet for each group and this advice was reinforced verbally. Both booklets were an A3 design (moderate-sucrose 12 pages, low-sucrose 14 pages). Both contained information on the causes of type 2 diabetes and importance of maintaining a healthy weight. Both gave examples of foods high in saturated fat, sources of hidden fats, low fat alternatives and advice on sensible alcohol consumption. Booklets differed in the advice given regarding sucrose. The low-sucrose group booklet warned of the potential impact of sucrose on glycaemic control and calorie intake. Advice was given to exclude or reduce sugar wherever possible e.g. to use reduced sugar jam; to avoid high sugar foods such as chocolate and confectioneries and to avoid adding sugar to foods and drinks. The moderate-sucrose group were permitted to include sugar at up to $10 \%$ of total daily energy intake and advised that moderate amounts of sugar could be included in their diet as part of a balanced diet. In practice this meant that this group were not explicitly advised to avoid sugar wherever possible e.g. to modify a recipe to reduce the sugar content. The only advice in the booklet for this group was to avoid highsugar drinks such as soft drinks. Further reinforcement of group-specific dietary advice was provided by the study nurse when participants attended for three monthly assessments.

\section{Food Diaries}

Participants were asked to record dietary intake over 3 consecutive days in food diaries at baseline and 3 monthly intervals for a period of 12 months. The version used was scaled down from that in the EPIC cancer study, and has been validated against food weighing (Bingham et al.
2001). The study dietitian gave participants instructions on how to complete the food diaries and estimate portion sizes using pictorial guides. The food diaries also contained detailed examples of how to describe and record foods.

\section{Statistical Analysis}

Specific outcome data from our own clinic were not available to perform a formal power calculation of patient numbers sufficient for the study. Based on previous short term studies that demonstrated significant effects of manipulating dietary sucrose, a sample of 76 (38 in each group) was estimated. Data were analysed using PASW statistics 18 (SPSS Inc, Chicago, IL). All data were checked for normality and log transformed where necessary to render their distribution normal before statistical analysis. These variables included LDL, HDL, and total cholesterol, TAG, HbA1c, glucose, HOMA-IR, BMI, body weight, total sugars $(\mathrm{g})$, and sucrose $(\mathrm{g} / \% \mathrm{E})$. A square root transformation was used to normalise the insulin data. Independent-samples ttests were used to examine whether there were any differences in the variables at baseline between the two dietary groups. Frequencies (\%), with Fisher's Exact test, were calculated for gender. Repeated measures ANOVA were performed on complete data sets to determine the effects of the low- and moderate-sucrose diets on anthropometric, biochemical and dietary variables, with 'visit' as the within-subject factor and 'diet' as the between-subjects factor. The total cholesterol: HDL ratio and alcohol intake data could not be normalised and so these data were analysed using nonparametric tests (Friedman test to determine the effect of visit and MannWhitney $U$ test to determine differences between diets). For significant main effects of 'visit', paired t-tests with Bonferroni correction were used to correct for multiple comparisons. In the group as a whole, Spearman's rank correlations determined the relationship between a) dietary intake and the anthropometric/biochemical variables at baseline, and b) change in dietary intake and change in the 
anthropometric/biochemical variables (12 months visit-baseline visit) over the study period. A Bonferroni correction was also applied to correct for multiple correlations. Values of $p \leq 0.05$ were taken as significant. Post hoc analysis was performed of the percent of under-reporting of energy intake compared to estimated energy requirements. Test meal TG levels over time were calculated using the trapezoid rule to measure area under the curve with no baseline $\left(\mathrm{AUC}_{\text {triglyceride }}\right)$ and incremental area under the curve with the fasting TG as baseline ( $\mathrm{iAUC}_{\text {triglyceride }}$ ).

Data from the food diaries on reported energy intake and macronutrient content of the diet were analysed using the nutritional analysis programme Dietplan 5.This uses the UK Nutrient Databank which comprises McCance \& Widdowson's $5^{\text {th }}$ Edition of 'The Composition of Foods' and was modified to enable more accurate recording of the sucrose content of the diet (McCance \&
Widdowson 2000). Macronutrients were presented as percentage contribution to total energy intake. The degree of over/under-reporting of energy intake was calculated using self-reported food diary intake and estimated energy requirements based on BMI at the corresponding clinic visit.

\section{Results}

41 participants were enrolled and 37 completed the study (low-sucrose group $\mathrm{n}=18$, moderate-sucrose group $\mathrm{n}=19$ ); 2 participants were withdrawn before randomisation after commencing gliclazide as only participants taking metformin monotherapy were included in the study. A third was lost to follow-up after the baseline visit (figure 1). A fourth participant was withdrawn due to commencing gliclazide after randomisation and has not been included in the statistical analysis.

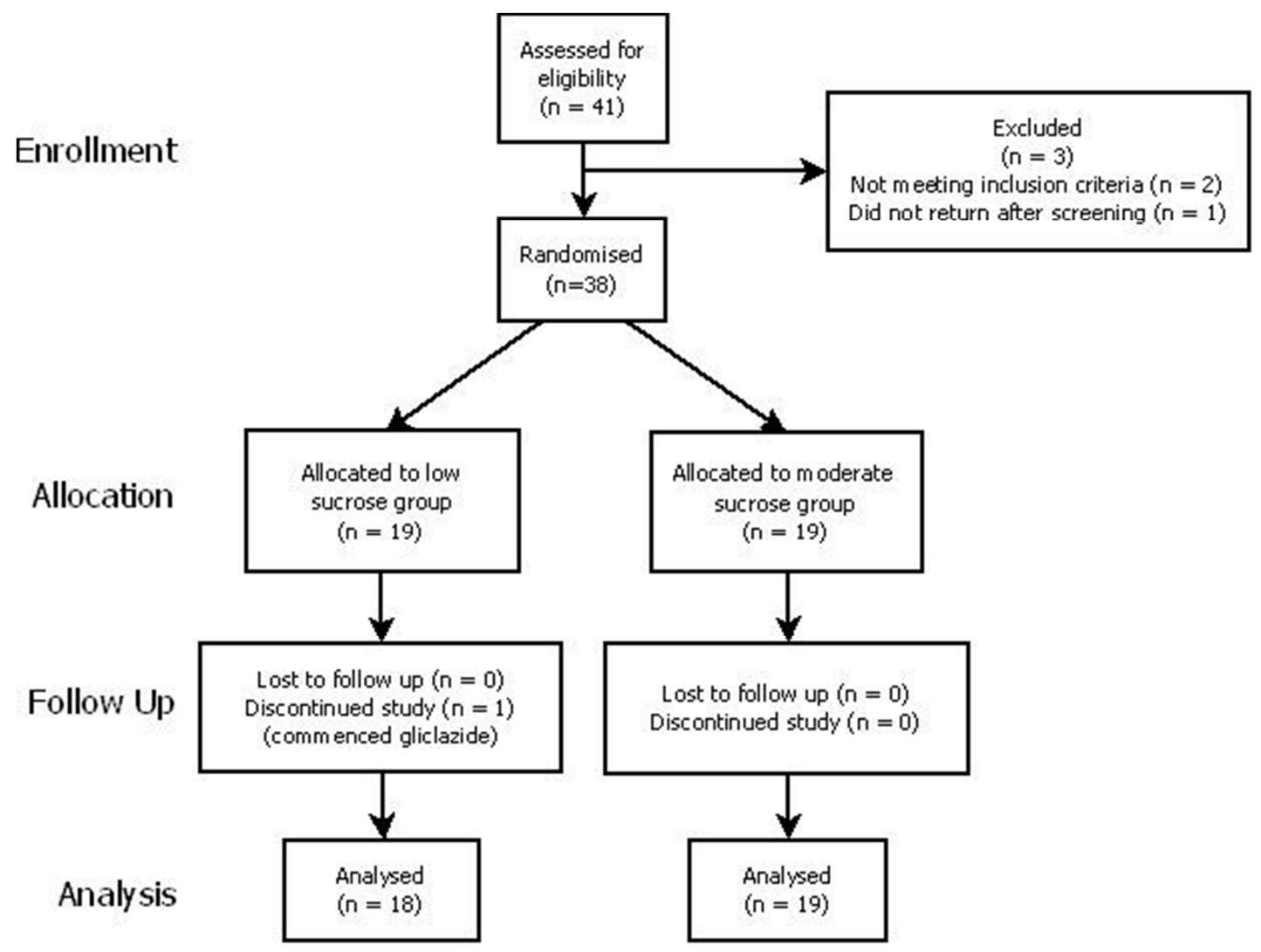

Figure 1. CONSORT Diagram Showing the Flow of Participants through Each Stage of the Study 


\section{Baseline Characteristics}

The groups were closely matched at baseline for age $(57.8 \pm 3.5 y$ rs low-sucrose, $58.2 \pm 3.0 \mathrm{yrs}$ moderate-sucrose) and

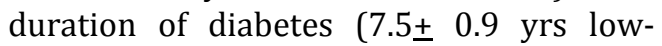

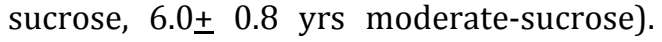
More males were noted in the moderatesucrose group than the low-sucrose group but differences were not significant $(63 \%$ vs $39 \%$ respectively).No significant baseline differences between groups were noted in anthropometric or metabolic variables (Table 1). Similarly, baseline dietary assessments showed no significant differences between the groups in their consumption of total energy, macronutrients, alcohol and sucrose. The test meal AUC triglyceride was significantly greater at baseline in the low-sucrose than moderate-sucrose group $(P=0.02)$. Both groups showed a gradual rise, reaching a peak at 3-4 hours and the start of a decline at 5 hours. No significant relationships between dietary and anthropometric/biochemical variables were noted at baseline after Bonferroni correction.

Table 1. Analysis of Anthropometric and Biochemical Variables Using Repeated

Measures ANOVA, with 'Visit' as the within-Subject Factor and 'Diet' as the betweenSubject Factor. Values Presented as Means \pm SEM. (Level of Bonferroni Correction 0.005)

\begin{tabular}{|c|c|c|c|c|c|c|c|c|}
\hline & \multicolumn{5}{|c|}{ Study visits } & \multirow[b]{2}{*}{$\begin{array}{l}P \\
\text { value } \\
\text { (inter } \\
\text { action } \\
\text { ) }\end{array}$} & \multirow[b]{2}{*}{$\begin{array}{l}\text { P value } \\
\text { (main } \\
\text { effects) }\end{array}$} & \multirow[b]{2}{*}{$\begin{array}{l}\text { P value } \\
\text { (Pairwise } \\
\text { comparis } \\
\text { ons) }^{1}\end{array}$} \\
\hline & $\begin{array}{l}\text { baselin } \\
\text { e }\end{array}$ & $\begin{array}{l}3 \\
\text { months }\end{array}$ & $\begin{array}{l}6 \\
\text { months }\end{array}$ & $\begin{array}{l}9 \\
\text { months }\end{array}$ & $\begin{array}{l}12 \\
\text { months }\end{array}$ & & & \\
\hline \multicolumn{9}{|c|}{ Anthropometrics } \\
\hline $\begin{array}{l}\text { Weight (kg) } \\
\operatorname{Low}(n=18) \\
\operatorname{Mod}(n=19)\end{array}$ & $\begin{array}{l}94.6 \pm \\
5.9 \\
91.0 \pm \\
3.2\end{array}$ & $\begin{array}{ll}93.0 \quad \pm \\
5.8 \\
89.8 \quad \pm \\
3.1\end{array}$ & $\begin{array}{ll}93.6 \quad \pm \\
5.9 \\
90.1 \quad \pm \\
3.1\end{array}$ & $\begin{array}{ll}93.2 \quad \pm \\
5.8 \\
90.6 \quad \pm \\
3.2\end{array}$ & $\begin{array}{ll}92.1 & \pm \\
5.6 & \\
90.6 \quad \pm \\
3.3 & \\
\end{array}$ & 0.15 & $0.01^{2}$ & $\mathrm{p}<0.001 \dagger$ \\
\hline $\begin{array}{c}\text { BMI }\left(\mathbf{k g} / \mathbf{m}^{2}\right) \\
\text { Low }(n=18) \\
\operatorname{Mod}(n=19)\end{array}$ & $\begin{array}{l}34.1 \pm \\
1.1 \\
32.4 \pm \\
0.9\end{array}$ & $\begin{array}{l}33.5 \pm \\
1.1 \\
32.0 \quad \pm \\
0.8 \\
\end{array}$ & $\begin{array}{ll}33.6 \quad \pm \\
1.1 \\
32.2 \quad \pm \\
0.8 \\
\end{array}$ & $\begin{array}{l}33.5 \quad \pm \\
1.1 \\
32.4 \quad \pm \\
0.8 \\
\end{array}$ & $\begin{array}{l}33.2 \pm \\
1.1 \\
32.4 \quad \pm \\
0.8\end{array}$ & 0.08 & $0.04^{2}$ & $\mathrm{p}<0.001 \dagger$ \\
\hline $\begin{array}{l}\text { Waist (cm) } \\
\text { Low }(n=18) \\
\operatorname{Mod}(n=19)\end{array}$ & $\begin{array}{l}109.3 \\
\pm 3.4 \\
107.9 \\
\pm 2.5 \\
\end{array}$ & $\begin{array}{l}109.1 \pm \\
4.3 \\
107.2 \pm \\
2.5\end{array}$ & $\begin{array}{l}110.1 \pm \\
4.3 \\
107.2 \pm \\
2.4\end{array}$ & $\begin{array}{l}109.2 \pm \\
4.2 \\
107.9 \pm \\
2.6\end{array}$ & $\begin{array}{l}107.4 \pm \\
4.1 \\
107.4 \pm \\
2.5\end{array}$ & 0.42 & & \\
\hline $\begin{array}{c}\text { Body fat (\%) } \\
\text { Low }(n=18) \\
\text { Mod }(n=19)\end{array}$ & $\begin{array}{l}40.4 \pm \\
1.6 \\
37.0 \pm \\
1.9\end{array}$ & $\begin{array}{ll}39.6 \quad \pm \\
1.5 \\
35.8 \quad \pm \\
1.7\end{array}$ & $\begin{array}{ll}40.3 \quad \pm \\
2.1 \\
36.8 \quad \pm \\
1.6 & \\
\end{array}$ & $\begin{array}{ll}38.3 \quad \pm \\
1.3 \\
36.2 \quad \pm \\
1.5\end{array}$ & $\begin{array}{ll}37.7 & \pm \\
1.4 & \\
36.2 & \pm \\
1.7 & \\
\end{array}$ & 0.31 & $0.04^{2}$ & \\
\hline \multicolumn{9}{|c|}{ Biochemical data } \\
\hline $\begin{array}{l}\text { Cholesterol } \\
(\mathbf{m m o l} / \mathbf{l}) \\
\operatorname{Low}(n=15) \\
\operatorname{Mod}(n=18)\end{array}$ & $\begin{array}{l}5.27 \pm \\
0.2 \\
5.14 \pm \\
0.2\end{array}$ & $\begin{array}{ll}5.29 & \pm \\
0.2 & \\
5.06 & \pm \\
0.2 & \\
\end{array}$ & $\begin{array}{ll}5.15 & \pm \\
0.3 & \\
5.16 \quad \pm \\
0.2 & \\
\end{array}$ & $\begin{array}{ll}5.08 & \pm \\
0.3 & \\
5.06 \quad \pm \\
0.2 & \\
\end{array}$ & $\begin{array}{ll}4.79 & \pm \\
0.2 & \\
5.03 & \pm \\
0.2 & \\
\end{array}$ & 0.45 & & \\
\hline $\begin{array}{c}\text { LDL (mmol/l) } \\
\operatorname{Low}(n=11) \\
\operatorname{Mod}(n=17)\end{array}$ & $\begin{array}{l}3.07 \pm \\
0.2 \\
3.01 \pm \\
0.2\end{array}$ & $\begin{array}{ll}3.11 & \pm \\
0.3 & \\
3.01 & \pm \\
0.2 & \\
\end{array}$ & $\begin{array}{ll}3.02 & \pm \\
0.3 & \\
3.01 & \pm \\
0.2 & \\
\end{array}$ & $\begin{array}{ll}2.78 \quad \pm \\
0.3 \\
2.92 \quad \pm \\
0.2 \\
\end{array}$ & $\begin{array}{ll}2.65 & \pm \\
0.1 & \\
2.98 & \pm \\
0.2 & \\
\end{array}$ & 0.40 & & \\
\hline $\begin{array}{c}\text { HDL (mmol/l) } \\
\text { Low }(n=14) \\
\operatorname{Mod}(n=18)\end{array}$ & $\begin{array}{l}1.28 \pm \\
0.1 \\
1.24 \pm\end{array}$ & $\begin{array}{ll}1.35 & \pm \\
0.1 & \\
1.27 & \pm \\
\end{array}$ & $\begin{array}{ll}1.52 & \pm \\
0.2 & \\
1.26 & \pm \\
\end{array}$ & $\begin{array}{ll}1.26 \pm \\
0.1 \\
1.27 \pm\end{array}$ & $\begin{array}{ll}1.22 & \pm \\
0.1 & \\
1.22 & \\
\end{array}$ & 0.21 & $0.05^{2}$ & $\mathrm{P}=0.002 \ddagger$ \\
\hline
\end{tabular}




\begin{tabular}{|c|c|c|c|c|c|c|c|c|}
\hline & 0.1 & 0.1 & 0.1 & 0.1 & 0.1 & & & \\
\hline $\begin{array}{l}\text { Ratio } \\
\text { Low }(n=13) \\
\text { Mod }(n=18)\end{array}$ & $\begin{array}{l}4.2 \pm \\
0.5 \\
4.2 \quad \pm \\
0.3\end{array}$ & $\begin{array}{ll}4.1 & \pm \\
0.3 & \\
4.1 & \pm \\
0.2 & \\
\end{array}$ & $\begin{array}{ll}3.9 & \pm \\
0.5 & \\
4.2 & \pm \\
0.2 & \\
\end{array}$ & $\begin{array}{l}4.2 \\
0.5 \\
4.2 \\
0.2\end{array}$ & $\begin{array}{ll}4.0 & \pm \\
0.4 & \\
4.2 & \pm \\
0.2 & \\
\end{array}$ & & & \\
\hline $\begin{array}{l}\text { Triglycerides } \\
\text { (mmol/l) } \\
\text { Low }(n=18) \\
\text { Mod }(n=19)\end{array}$ & $\begin{array}{l}2.25 \pm \\
0.3 \\
1.92 \pm \\
0.2 \\
\end{array}$ & $\begin{array}{ll}2.17 & \pm \\
0.2 & \\
1.90 \quad \pm \\
0.3\end{array}$ & $\begin{array}{ll}2.10 & \pm \\
0.3 & \\
1.89 & \pm \\
0.2 & \\
\end{array}$ & $\begin{array}{ll}2.52 & \pm \\
0.4 & \\
2.00 & \pm \\
0.3 & \\
\end{array}$ & $\begin{array}{ll}1.98 & \pm \\
0.2 & \\
1.96 & \pm \\
0.3 & \\
\end{array}$ & 0.50 & & \\
\hline $\begin{array}{c}\text { HbA1c (\%) } \\
\text { Low }(n=18) \\
\text { Mod }(n=19)\end{array}$ & $\begin{array}{l}7.7 \pm \\
0.3 \\
7.6 \quad \pm \\
0.3\end{array}$ & $\begin{array}{l}7.6 \pm 0.2 \\
7.4 \quad \pm \\
0.3\end{array}$ & $\begin{array}{ll}7.4 & \pm \\
0.2 & \\
7.4 & \pm \\
0.3 & \end{array}$ & $\begin{array}{ll}7.3 & \pm \\
0.3 & \\
7.5 & \pm \\
0.3 & \end{array}$ & $\begin{array}{ll}7.1 & \pm \\
0.2 & \\
7.4 & \pm \\
0.2 & \end{array}$ & 0.21 & $0.003^{2}$ & $\begin{array}{l}\mathrm{p}<0.001 \dagger \\
\dagger\end{array}$ \\
\hline $\begin{array}{l}\text { Glucose } \\
(\mathbf{m m o l} / \mathbf{l}) \\
\text { Low }(n=14) \\
\operatorname{Mod}(n=17)\end{array}$ & $\begin{array}{l}8.71 \pm \\
0.7 \\
8.27 \pm \\
0.5 \\
\end{array}$ & $\begin{array}{ll}8.84 \quad \pm \\
0.8 \\
8.31 \quad \pm \\
0.7\end{array}$ & $\begin{array}{ll}7.76 & \pm \\
0.5 & \\
8.12 & \pm \\
0.4 & \\
\end{array}$ & $\begin{array}{ll}7.96 & \pm \\
0.5 & \\
8.42 & \pm \\
0.5 & \\
\end{array}$ & $\begin{array}{ll}7.64 \quad \pm \\
0.5 \\
7.84 \quad \pm \\
0.4 & \\
\end{array}$ & 0.27 & & \\
\hline $\begin{array}{c}\text { Insulin (mu/l) } \\
\operatorname{Low}(n=12) \\
\operatorname{Mod}(n=17)\end{array}$ & $\begin{array}{l}11.8 \pm \\
1.0 \\
13.7 \pm \\
2.0\end{array}$ & $\begin{array}{l}10.9 \pm \\
1.3 \\
13.9 \pm \\
1.8\end{array}$ & $\begin{array}{ll}10.2 & \pm \\
1.0 & \\
15.1 & \pm \\
2.3 & \end{array}$ & $\begin{array}{l}10.4 \pm \\
1.0 \\
15.0 \pm \\
1.6\end{array}$ & $\begin{array}{ll}9.2 & \pm \\
1.0 & \\
14.1 & \pm \\
1.9 & \end{array}$ & 0.14 & & \\
\hline \multicolumn{9}{|l|}{ Test meal } \\
\hline $\begin{array}{l}\text { AUC triglyceride } \\
\text { Low }(n=10) \\
\text { Mod }(n=12)\end{array}$ & $\begin{array}{l}1020 \\
\pm \\
134^{\dagger \dagger} \\
652 \pm \\
69\end{array}$ & & & & $\begin{array}{ll}926 & \pm \\
148 & \\
689 & \pm \\
82 & \end{array}$ & 0.01 & $0.05^{3}$ & \\
\hline $\begin{array}{l}\text { IAUC triglyceride } \\
\text { Low }(n=10) \\
\operatorname{Mod}(n=12)\end{array}$ & $\begin{array}{l}219 \pm \\
44 \\
152 \pm \\
33\end{array}$ & & & & $\begin{array}{ll}218 & \pm \\
58 & \\
179 & \pm \\
32 & \end{array}$ & 0.16 & & \\
\hline
\end{tabular}

1 significant $\mathrm{p}$ values only, ${ }^{2}$ visit $^{3}$ diet

† baseline vs 3 months

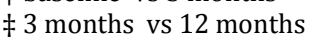

††baseline vs 12 months

${ }^{+} p=0.02$, compared to the moderate-sucrose group V1.

\section{Anthropometric Outcomes}

Bodyweight, BMI and \% body fat all decreased during the study $(\mathrm{P} \leq 0.042)$, with no effect of dietary group (Table 1). Although weight loss was moderate (1$2 \mathrm{~kg}$ ), it was maintained in both groups throughout the study.

\section{Metabolic Outcomes}

HbA1c concentrations decreased throughout the study $(\mathrm{P}=0.003)$ with no effect of dietary group, falling from $7.7 \pm$ $0.3 \%$ at baseline to $7.1 \pm 0.2 \%$ at 12 months in the low-sucrose group and $7.6 \pm$
$0.3 \%$ to $7.4 \pm 0.2 \%$ in the moderatesucrose group (Table 1). Whilst there was no significant change in fasting glucose and no effect of dietary group, a marked, but non-significant reduction was noted between the baseline and 12 month visits, particularly in the low-sucrose group (8.7 \pm $0.7 \mathrm{mmol} / \mathrm{l}$ to $7.6 \pm 0.5 \mathrm{mmol} / \mathrm{l}$ ).

A significant visit*diet interaction was noted for HOMA-IR $(\mathrm{p}=0.009)$ (Figure 2). Whilst HOMA-IR tended to increase in the moderate sucrose group, posthoc analysis revealed that for the low sucrose group, HOMA-IR reduced significantly at 3 months and at 12 months compared with baseline 
$(p \leq 0.005)$. No significant within- or between-group differences were seen in lipid measures apart from HDL concentrations which varied over time $(\mathrm{P}=0.048)$, being significantly lower at the end of study than at 3 months $(P=0.002)$.
The profiles of triglyceride increases above the pre-test value ( $\Delta$ triglyceride) showed no significant group differences at any time, nor was there any difference in iAUC $_{\text {triglyceride }}$

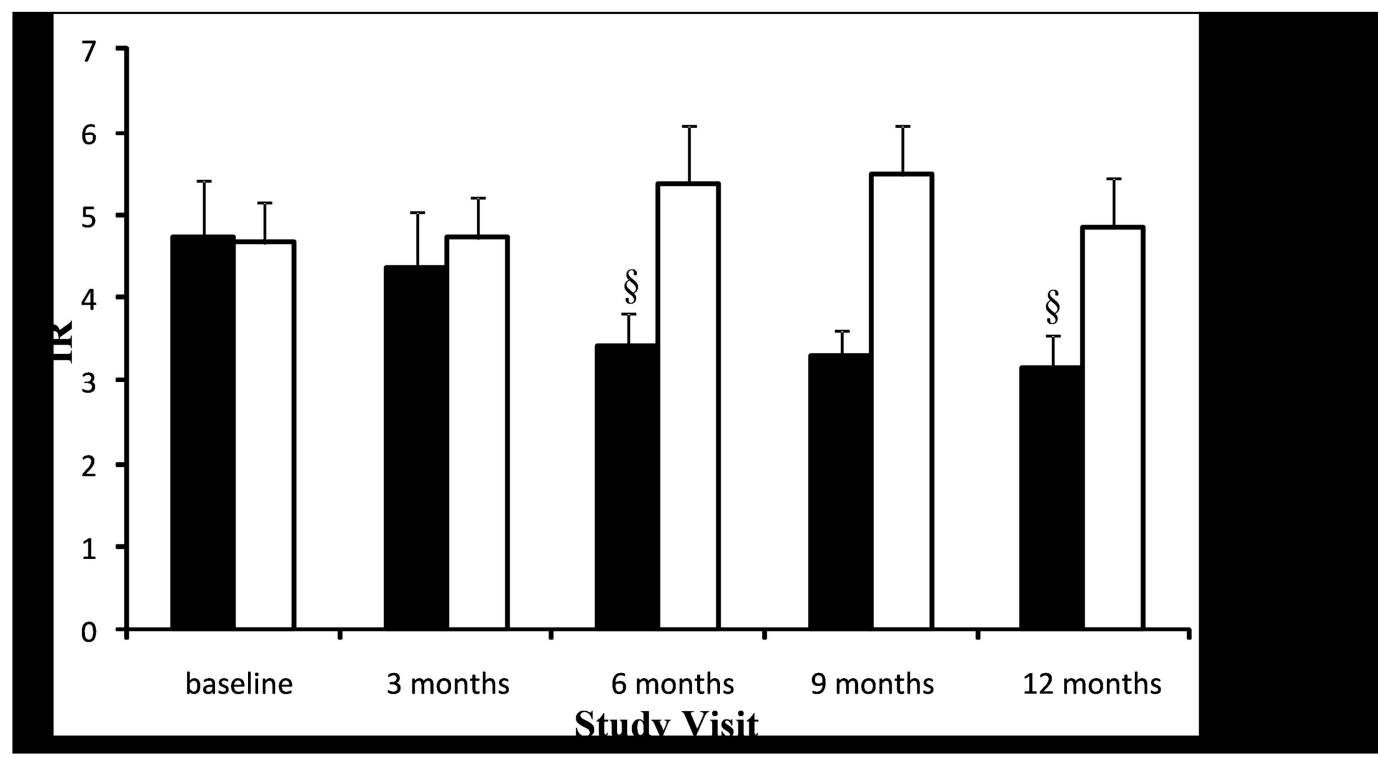

Figure 2: Analysis of HOMA-IR in the Low-sucrose ( $n=10$, black bars) and ModerateSucrose (n=16, white bars) Groups at Baseline- 12 Month Visits Using Repeated

\section{Dietary Outcomes}

Significant reductions in self-reported energy intake were noted during the study ( $\mathrm{p}<0.001)$, with no difference by dietary group (Table 2). Participants in both groups under-reported energy intake compared to estimated energy requirements by, on average, 25\% (28\% low-sucrose vs $22 \%$ moderate-sucrose). Within-group differences in underreporting were significant at every time point except at 3 months (low-sucrose group) and at 12 months (moderatesucrose group). 
Table 2. Analysis of Dietary Variables Using Repeated Measures ANOVA, with 'Visit' as the within-Subject Factor and 'Diet' as the between-Subject Factor. Values Presented as Means \pm SEM,

\begin{tabular}{|c|c|c|c|c|c|c|}
\hline & & $\begin{array}{l}\text { Study } \\
\text { visits }\end{array}$ & & & & \multirow{2}{*}{$\begin{array}{l}\text { P value } \\
\text { (Pairwise } \\
\text { comparisons) }\end{array}$} \\
\hline & baseline & 3 months & $\begin{array}{l}12 \\
\text { months }\end{array}$ & $\begin{array}{l}\text { P value } \\
\text { (Interaction) }\end{array}$ & $\begin{array}{c}P \\
\text { value } \\
\quad \text { (Visit) }\end{array}$ & \\
\hline $\begin{array}{l}\text { Total Energy (MJ) } \\
\text { Low }(n=11) \\
\text { Mod }(n=14)\end{array}$ & $\begin{array}{l}9.1 \pm 1.0 \\
9.0 \pm 0.6\end{array}$ & $\begin{array}{l}7.6 \pm 0.8 \\
7.1 \pm 0.4\end{array}$ & $\begin{array}{l}6.9 \pm 0.4 \\
7.2 \pm 0.4\end{array}$ & 0.63 & $<\mathbf{0 . 0 0 1}^{2}$ & $\dagger p<0.001$ \\
\hline $\begin{array}{l}\text { Protein }(\% \mathbf{E}) \\
\text { Low }(\mathrm{n}=11) \\
\operatorname{Mod}(\mathrm{n}=14)\end{array}$ & $\begin{array}{l}18.3 \pm 1.2 \\
18.4 \pm 0.8\end{array}$ & $\begin{array}{l}17.7 \pm 0.8 \\
20.9 \pm 0.8\end{array}$ & $\begin{array}{ll}20.3 & \pm \\
0.9 & \\
20.3 & \pm \\
1.1 & \\
\end{array}$ & 0.06 & $0.04^{2}$ & $\ddagger p=0.017$ \\
\hline $\begin{array}{l}\text { Fat }(\% \mathbf{E}) \\
\text { Low }(n=11) \\
\operatorname{Mod}(n=14)\end{array}$ & $\begin{array}{l}34.9 \pm 1.1 \\
36.1 \pm 2.2\end{array}$ & $\begin{array}{l}31.2 \pm 2.2 \\
31.5 \pm 1.6\end{array}$ & $\begin{array}{ll}29.3 & \pm \\
1.1 & \\
27.4 & \pm \\
1.6 & \\
\end{array}$ & 0.48 & $<0.001^{2}$ & $\ddagger p<0.001$ \\
\hline $\begin{array}{l}\text { Carbohydrate }{ }^{3} \quad(\% \\
\text { E) } \\
\text { Low }(n=11) \\
\text { Mod }(n=14)\end{array}$ & $\begin{array}{l}43.9 \pm 1.1 \\
44.6 \pm 1.8\end{array}$ & $\begin{array}{l}47.2 \pm 2.5 \\
46.3 \pm 1.6\end{array}$ & $\begin{array}{ll}47.2 & \pm \\
1.7 & \\
52.3 & \pm \\
1.6 & \\
\end{array}$ & 0.07 & $<\mathbf{0 . 0 0 1}^{2}$ & $\ddagger \mathrm{p}<0.001$ \\
\hline $\begin{array}{l}\text { Alcohol (\% E) } \\
\text { Low (n=11) } \\
\text { Mod (n=14) }\end{array}$ & $\begin{array}{l}2.96 \pm 1.2 \\
1.04 \pm 0.5\end{array}$ & $\begin{array}{l}3.95 \pm 1.8 \\
1.21 \pm 0.9\end{array}$ & $\begin{array}{ll}3.25 & \pm \\
1.5 & \\
1.14 & \pm \\
0.6 & \\
\end{array}$ & & & \\
\hline $\begin{array}{c}\text { Sucrose (\% E) } \\
\text { Low }(n=11) \\
\operatorname{Mod}(n=14)\end{array}$ & $\begin{array}{l}5.2 \pm 0.8 \\
4.7 \pm 0.7\end{array}$ & $\begin{array}{l}6.7 \pm 1.2 \\
4.3 \pm 0.6\end{array}$ & $\begin{array}{l}6.2 \pm 0.8 \\
5.1 \pm 0.5\end{array}$ & 0.25 & & \\
\hline $\begin{array}{l}\text { Sucrose (g) } \\
\text { Low }(n=11) \\
\operatorname{Mod}(n=14)\end{array}$ & $\begin{array}{l}25.7 \pm 3.8 \\
27.2 \pm 6.0\end{array}$ & $\begin{array}{l}26.6 \pm 3.9 \\
18.5 \pm 3.0\end{array}$ & $\begin{array}{ll}25.2 & \pm \\
3.8 & \\
21.8 & \pm \\
2.6 & \end{array}$ & 0.34 & & \\
\hline $\begin{array}{l}\text { Total sugars }^{4} \\
\text { Low }(n=11) \\
\text { Mod }(n=14)\end{array}$ & $\begin{array}{l}75.6 \pm 9.3 \\
75.5 \\
10.5\end{array}$ & $\begin{array}{l}80.6 \pm 8.7 \\
61.0 \pm 6.7\end{array}$ & $\begin{array}{ll}69.2 & \pm \\
7.1 & \\
71.4 & \pm \\
6.5 & \end{array}$ & 0.10 & & \\
\hline $\begin{array}{l}\text { Total sugars }(\% \mathbf{E}) \\
\text { Low }(n=11) \\
\operatorname{Mod}(n=14)\end{array}$ & $\begin{array}{l}15.0 \pm 1.9 \\
13.7 \pm 1.3\end{array}$ & $\begin{array}{l}18.9 \pm 2.2 \\
14.3 \pm 1.2\end{array}$ & $\begin{array}{ll}17.0 & \pm \\
1.5 & \\
16.5 & \pm \\
1.1 & \\
\end{array}$ & 0.52 & $\mathrm{0.03}^{1}$ & \\
\hline $\begin{array}{ll}\text { (\%) } & \text { under- } \\
\text { reporting } 5 & \\
\end{array}$ & & & & & & \\
\hline Low $(\mathrm{n}=18)$ & 9 & 25 & $28^{*}$ & & & \\
\hline $\operatorname{Mod}(n=18)$ & 10 & $29^{*}$ & 22 & & & \\
\hline
\end{tabular}

${ }^{1}$ significant $\mathrm{p}$ values only ${ }^{2}$ visit, ${ }^{3}$ combination of starch and sugars ${ }^{4}$ natural and added sugars, ${ }^{5}$ Number of completed food diaries varies between time points

† baseline vs 12 months, baseline vs 3 months,

‡ baseline vs 12 months

$* \mathrm{p}<0.01$

There were significant increases in both carbohydrate and protein intake, and a significant decrease in fat (all $\mathrm{p}<0.001$ ), with no differences found between dietary groups.. Both groups reported low sucrose intakes at baseline and appeared to reduce 
their sucrose intake even further as the study progressed. Within-and betweengroup differences in sucrose intakes were not statistically significant.

\section{Relationships between Dietary Intake and Anthropometric/Biochemical Measures}

In the group as a whole, no correlations were found between changes (baseline-12 months) in dietary and anthropometric/biochemical variables. When data were split by dietary group, the only correlation observed in the low sucrose group after Bonferroni correction was a negative correlation between LDL and sucrose $\% \mathrm{E}(\mathrm{r}=-0.823, \mathrm{p}=0.003)$. In the moderate-sucrose group, positive correlations were noted between reductions in blood glucose and energy intake $(\mathrm{r}=0.772, \mathrm{p}=0.001)$ and blood glucose and fat intake ( $r=0.693, p=0.002)$. The relationship between waist circumference (which remained virtually unchanged throughout the study) and reduction in fat intake was also significant $(\mathrm{r}=0.712, \mathrm{p}=0.004)$.

\section{Discussion}

The most important finding of this study is that giving advice to relax restrictions on sucrose intake to individuals with type 2 diabetes had little effect on reported behaviour, metabolic control or biochemical responses to test meals. We had hypothesised that emphasising a single dietary message, as opposed to a dual message would improve compliance and allow participants to focus on eating less fat, thereby reducing total energy intake. This in turn would be reflected in a reduction in body weight and fat mass, improved insulin sensitivity, lower blood glucose and lower HbA1c, without causing any deterioration in lipids and triglycerides.

Although both groups saw significant reductions from baseline in $\mathrm{HbA1c}$, weight and body fat, the primary and secondary endpoints of significantly greater reductions in HbA1c and weight in the moderate-sucrose group were not achieved. On a positive note, deterioration in lipids and triglyceride levels were not observed. It is possible that the lack of significant differences between groups in weight and HbA1c are due to a Type II error resulting from a small sample size. Although a sample size of 76 was originally estimated to be required, recruitment was slower than anticipated, resulting in 37 evaluable participants. Retrospective power calculation analysis suggests this sample size has only a $40 \%$ power to detect a clinically significant difference of $0.7 \%$ in HbA1c levels between groups at 12 months. The trends seen in this study could be considered to favour the low-sucrose diet as opposed to the moderate sucrose diet, however a much larger study would be needed to establish the true effect of these two dietary approaches to diabetes management.'

Although a significant group-diet interaction in HOMA-IR levels was noted with significantly improved levels over time in the low-sucrose group and a trend for levels to increase in the moderatesucrose group, between group differences at 12 months were not significant .Again this, may be accounted for by sample size. As ANOVA only computes complete data sets, the HOMA-IR analysis is based on a relatively small sample size of 10 lowsucrose participants and 16 moderatesucrose participants. Retrospective power calculation analysis suggests a minimal sample size of 23 would be required to detect a clinically significant difference of 1.96 in HOMA-IR levels between groups at 12 months. Reasons for the improvement in insulin sensitivity in the low-sucrose group as opposed to the moderate-sucrose group are unclear as participants did not report meaningful changes in dietary habits with regards to sucrose intake and both groups reported increases in carbohydrate intake and reductions in fat. Despite an increase in protein intake in the low-sucrose group during the study, no significant between group differences were noted.

Evidence from the food diaries suggested participants were able to focus on consuming a low fat diet and to reduce 
their total energy intake. Similar levels of compliance were seen in both groups and may be because both groups reported low sucrose intakes at baseline, in keeping with entrenched dietary advice to avoid sucrose. The moderate-sucrose group in particular appeared confused with guidelines to change sucrose habits. Despite advice to the contrary, this group actually reported greater, though non-significant reductions in sucrose intake at 12 months than the low-sucrose group. Best practice would require analysis of food diaries to be undertaken independently and not by study researchers. Caution must be taken therefore in interpreting dietary findings as this lack of independent analysis could potentially introduce bias.

Reported energy intakes were lower than baseline in both groups at all time points.

Greater weight loss may have been anticipated, given the reported fall in energy intake. Assessment of diet in epidemiological studies is often based on self-reported methods and is dependent on the accuracy with which participants record their dietary intake. During this study period, some participants had a tendency to avoid completing diaries as the study progressed and some degree of under-reporting was also noted.

Under-reporting, particularly of energy intake, is a common phenomenon and is more likely in overweight and obese individuals (women more so than men) compared with their lean counterparts. As Blundell (2000) and Goris et al (2000) suggest, underreporting may be less about the ability to comply with instructions, than the emotional and moral feelings that influence the perceived value of making a true record of events. Consideration of under-reporting is therefore important when examining associations between energy intake, macro and micro nutrient composition of diets. Evidence suggests that the degree of under-reporting of energy intake in epidemiological studies ranges from as low as 10\% (Garriguet 2008 ) to as high as $47 \%$ (Lichtman et al. 1992). More akin to the population of this study, in Rennie, Coward \& Jebb's (2007)
UK-based study of the 2000 National Diet and Nutrition Survey, underreporting of energy intake was calculated to be $25 \%$.This suggests our results are representative of large-scale epidemiological trials. The trend towards a greater degree of under-reporting in the low-sucrose group may have been a consequence of receiving more severe restrictive advice.

It is worth noting that weight fell across both groups during the course of the intervention, although, at each time point total energy expenditure was recalculated using the most recent weight, and as such the difference in energy intake versus energy expenditure cannot be explained by weight loss. We believe the reported dietary intake reflects dietary reporting in large scale epidemiological studies, However it is impossible to establish the degree of underreporting of any specific macronutrient group such as sucrose, especially given the ingrained negative perception of sugar/sucrose among individuals with diabetes. Furthermore, we know that reported energy intakes fell over the course of the study. Many foods that are high in fat, such as chocolate, sweet pastries and puddings also have a tendency to have a high sugar content. It is possible that when people are given the instruction to reduce fat intake, sucrose content unintentionally falls as well.

Lack of contact with an appropriate professional may also have influenced the outcome of the study. Participants met a dietitian once only at the start of the study and the advice was reinforced by the study nurse at subsequent visits. Whilst this design was intended to replicate the activity in a typical outpatient clinic, it must be acknowledged that frequent contact with a dietitian has been shown to have a positive effect on dietary change in individuals with type 2 diabetes (Franz et al. 1995).

In addition, it is possible that the use of a booklet, which could be considered a 'prescriptive' approach to reinforce new dietary messages is not a strong enough method to change established behaviour. 
Individuals are more likely to respond to positively framed messages in which they have an understanding of the benefits of an action as opposed to the adverse consequences (van Assemana et al. 2001). As Moran (2004) suggests, this is best delivered using structured education techniques and ongoing support to facilitate lasting behaviour change.

Whilst it is generally acknowledged that individuals find positive dietary messages (such as eat 5 items of fruit and vegetables a day) less confusing than negative messages; individuals with established type 2 diabetes have traditionally tended to concentrate on the negative message of avoiding sucrose, often at the expense of other dietary advice and have incorporated this into their lifestyle since the early days of diagnosis. These results show that advice to relax this restriction was ignored in this study as it would require considerable changes to habit and might be viewed as lacking in credibility and reliability (Kasila et al. 2003; Kelly \& Stanner 2003). The challenge remains as to how best to encourage individuals with established type 2 diabetes to 'unlearn' previous dietary messages and join newly diagnosed individuals in making fat restriction the primary focus of their dietary endeavours.

\section{Acknowledgements}

The study team would like to express gratitude to the individuals who volunteered to take part in this study. The team would particularly like to thank Professor Gareth Williams for his considerable input in the design and management of this study. This study was supported by a grant from Sugar Nutrition UK (formerly The Sugar Bureau). The authors have no other relevant conflict of interest to declare.

\section{References}

Abraira, C. \& Derler, J. (1988). "Large Variations of Sucrose in Constant Carbohydrate Diets in Type II Diabetes," The American Journal of Medicine, 84 ( 2) 193-200.
Avignon, A., Radauceanu, A. \& Monnier, L. (1997). "Nonfasting Plasma Glucose is a Better Marker of Diabetic Control than Fasting Plasma Glucose in Type 2 Diabetes," Diabetes Care, 20 (12)18221826.

Bantle, J. P., Laine, D. C. \& Thomas, J. W. (1986). "Metabolic Effects of Dietary Fructose and Sucrose in Types I and II Diabetic Subjects," JAMA, 256(23) 32413246.

Bingham, S. A., Welch, A. A., McTaggart, A., Mulligan, A. A., Runswick, S. A., Luben, R., Oakes, S., Khaw, K. T., Wareham, N. \& Day, N. E. (2001). "Nutritional Methods in the European Prospective Investigation of Cancer in Norfolk," Public Health Nutrition, 4 (3) 847-858.

Black, R. N. A., Spence, M., McMahon, R. O., Cuskelly, G. J., Ennis, C. N., McCance, D. R., Young, I. S., Bell, P. M. \& Hunter, S. J. (2006). "Effect of Eucaloric High- and Low-Sucrose Diets with Identical Macronutrient Profile on Insulin Resistance and Vascular Risk: A Randomized Controlled Trial," Diabetes, 55 (12) 3566-3572.

Blundell, J. E. (2000). "What Foods Do People Habitually Eat? A Dilemma for Nutrition, an Enigma for Psychology," The American Journal of Clinical Nutrition, 71 (1) 3-5.

Department of Health (1991). Dietary Reference Values for Food Energy and Nutrients for the UK. Report of the Panel on Dietary Reference Values of the Committee on Medical Aspects of Food Policy, HMSO, London. Dietplan5. Forest Field Software limited.[Retrieved January 22,2014], http://www.forestfield.co.uk/Dietplan5/di etplan5.html.

Dyson, P. A., Kelly, T., Deakin, T., Duncan, A., Frost, G., Harrison, Z., Khatri, D., Kunka, D., Mcardle, P., Mellor, D., Oliver, L. \& Worth, J. (2011). "Diabetes UK Evidence-Based Nutrition Guidelines for the Prevention and Management of Diabetes," Diabetic Medicine. 28(11) 1282-1288. 
Franz, M. J., Monk, A., Barry, B., McClain, K., Weaver, T., Cooper, N., Upham, P., Bergenstal, R. \& Mazze, R. S. (1995). "Effectiveness of Medical Nutrition Therapy Provided by Dietitians in the Management of Non-Insulin-Dependent Diabetes Mellitus: A Randomized, Controlled Clinical Trial," Journal of the American Dietetic Association, 95 (9) 1009-1017.

Garriguet, D. (2008). "Under-Reporting of Energy Intake in the Canadian Community Health Survey," Health Information and Research Division, 19 (4) 37-45.

Goris, A. H., Westerterp-Plantenga, M. S. \& Westerterp, K. R. (2000). "Undereating and Underrecording of Habitual Food Intake in Obese Men: Selective Underreporting of Fat Intake," The American Journal of Clinical Nutritio, 71(1) 130-134.

Jebb, S. A., Cole, T. J., Doman, D., Murgatroyd, P. R. \& Prentice, A. M. (2000). "Evaluation of the Novel Tanita Body-Fat Analyser to Measure Body Composition by Comparison with a Four-Compartment Model," British Journal of Nutrition, 83(2) 115-122.

Jellish, W. S., Emanuele, M. A. \& Abraira, C. (1984). "Graded Sucrose/Carbohydrate Diets in Overtly Hypertriglyceridemic Diabetic Patients," The American Journal of Medicine, 77(6) 1015-1022.

Kasila, K., Poskiparta, M., Karhila, P. \& Kettunen, T. (2003). "Patients' Readiness for Dietary Change at the Beginning of Counselling: A Transtheoretical ModelBased Assessment," Journal of Human Nutrition and Dietetics, 16(3)159-166.

Kelly, C. N. M. \& Stanner, S. A. (2003). "Diet and Cardiovascular Disease in the UK: Are the Messages Getting Across?," Proceedings of the Nutrition Society, 62(3) 583-589.

Lichtman, S. W., Pisarska, K., Berman, E. R., Pestone, M., Dowling, H., Offenbacher, E., Weisel, H., Heshka, S., Matthews, D. E. \& Heymsfield, S. B. (1992). "Discrepancy between Self-Reported and Actual Caloric Intake and Exercise in Obese Subjects," The
New England Journal of Medicine, 327 (27) 1893-1898.

Matthews, D. R., Hosker, J. P., Rudenski, A. S., Naylor, B. A., Treacher, D. F. \& Turner, R. C. (1985). "Homeostasis Model Assessment: Insulin Resistance and BetaCell Function from Fasting Plasma Glucose and Insulin Concentrations in Man," Diabetologia, 28(7) 412-419.

McCance, R. \& Widdowson, E. (2000). 'The Composition of Foods. The Royal Society of Chemistry,' Cambridge and the Ministry of Agriculture, Fisheries and Food, London.

Mensah, G. A., Mokdad, A. H., Ford, E., Narayan, K. M., Giles, W. H., Vinicor, F. \& Deedwania, P. C. (2004). "Obesity, Metabolic Syndrome, and Type 2 Diabetes: Emerging Epidemics and Their Cardiovascular Implications," Cardiology Clinics, 22 (4) 485-504.

Mora, S., Rifai, N., Buring, J. E. \& Ridker, P. M. (2008). "Fasting Compared with Nonfasting Lipids and Apolipoproteins for Predicting Incident Cardiovascular Events," Circulation, 118(10) 993-1001.

Moran, M. (2004). "The Evolution of the Nutritional Management of Diabetes," Proceedings of the Nutrition Society, 63(4) 615-620.

Rennie, K. L., Coward, A. \& Jebb, S. A. (2007). "Estimating Under-Reporting of Energy Intake in Dietary Surveys Using an Individualised Method," British Journal of Nutrition, 97 (6) 1169-1176.

Riserus, U., Willett, W. C. \& Hu, F. B. (2009). "Dietary Fats and Prevention of Type 2 Diabetes," Progress in Lipid Research, 48 (1) 44-51.

Schofield, W. N. (1985). "Predicting Basal Metabolic Rate, New Standards and Review of Previous Work," Human Nutrition. Clinical Nutrition, 39 (Suppl 1) 5-41.

Shaw, J. E., Sicree, R. A. \& Zimmet, P. Z. (2010). "Global Estimates of the Prevalence of Diabetes for 2010 and 2030," Diabetes Research and Clinical Practice, 87(1) 4-14. 
Smyth, S. \& Heron, A. (2006). "Diabetes and Obesity: The Twin Epidemics," Nature Medicine, 12(1) 75-80.

Van Assema, P., Martens, M., Ruiter, R. A. C. \& Brug, J. (2001). "Framing of Nutrition Education Messages in Persuading Consumers of the Advantages of a Healthy Diet," Journal of Human Nutrition and Dietetics, 14, (6) 435-442.
Van Oostrom, A. J., Alipour, A., Sijmonsma, T. P., Verseyden, C., Linga-Thie, G. M., Plokker, H. W. \& Castro, C. M. (2009). "Comparison of Different Methods to Investigate Postprandial Lipaemia," The Netherlands Journal of Medicine 67(1) 1320. 